\title{
A new setup for the quantitative analysis of drying by the use of gas-phase FTIR- spectroscopy
}

F. Scheepers, A. Staehler, M. Staehler, M. Carmo, W. Lehnert, and D. Stolten

Citation: Review of Scientific Instruments 89, 083102 (2018); doi: 10.1063/1.5036817

View online: https://doi.org/10.1063/1.5036817

View Table of Contents: http://aip.scitation.org/toc/rsi/89/8

Published by the American Institute of Physics

\section{Articles you may be interested in}

A practical wide-field Raman imaging method with high spectral and spatial resolution

Review of Scientific Instruments 89, 083103 (2018); 10.1063/1.5041529

Printed-circuit-board linear Paul trap for manipulating single nano- and microparticles

Review of Scientific Instruments 89, 083101 (2018); 10.1063/1.5007924

Time base evaluation for atom gravimeters

Review of Scientific Instruments 89, 083104 (2018); 10.1063/1.5039653

A sensitive, low noise, DC to $12 \mathrm{MHz}$, large area photodiode preamplifier for photothermal heterodyne imaging

Review of Scientific Instruments 89, 083105 (2018); 10.1063/1.5036626

Invited Review Article: Modeling ion beam extraction from different types of ion sources

Review of Scientific Instruments 89, 081101 (2018); 10.1063/1.5002001

Surface action spectroscopy with rare gas messenger atoms

Review of Scientific Instruments 89, 083107 (2018); 10.1063/1.5045324

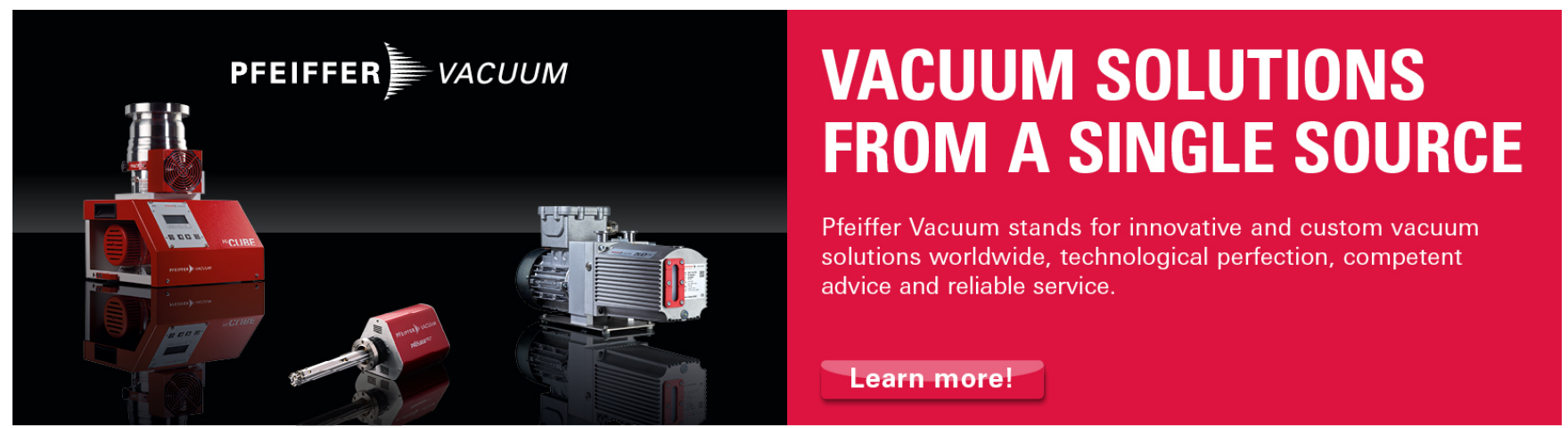




\title{
A new setup for the quantitative analysis of drying by the use of gas-phase FTIR-spectroscopy
}

\author{
F. Scheepers, ${ }^{1}$ A. Staehler, ${ }^{1}$ M. Staehler, ${ }^{1}$ M. Carmo, ${ }^{1}$ W. Lehnert,,${ }^{1,2}$ and D. Stolten ${ }^{1,2}$ \\ ${ }^{1}$ Institute of Energy and Climate Research IEK3, Forschungszentrum Juelich GmbH, 52425 Juelich, Germany \\ ${ }^{2}$ Faculty of Mechanical Engineering, RWTH Aachen, 52062 Aachen, Germany
}

(Received 19 April 2018; accepted 5 July 2018; published online 3 August 2018)

\begin{abstract}
Drying rates are important for the manufacture of thin films and in specific for the production of electrodes used in electrochemical devices such as fuel cells and electrolyzers. The known procedures to investigate time-dependent sample compositions and selective evaporation rates are insufficient to obtain mean information about the full area instead of a single point analysis. Therefore, a new setup is presented using gas-phase Fourier-transform infrared spectroscopy. This method analyzes the gas-phase composition to recalculate the layer composition in electrode fabrication at any time during drying. According to the golden rule of measurement technology, manufacturer specifications are often overestimated. Therefore, our alternative procedures were used to evaluate the precision of devices used. The calculated measurement precision is confirmed by validation. The expected deviation is quantified to be less than $2 \%$ for the common application. Further on, the relative testretest standard deviation is determined to be $0.3 \%-0.4 \%$. As a result of the error propagation, the measurement precision is limited by the background gas flow rate precision for common application. At low volume fractions, the influence of the substance flow rate deviations becomes significant. However, further studies will focus on increasing the gas flow rate precision. Published by AIP Publishing. https://doi.org/10.1063/1.5036817
\end{abstract}

\section{INTRODUCTION}

In many technical sectors like battery, ${ }^{1}$ photovoltaic, ${ }^{2}$ pharmaceutical, ${ }^{3}$ and food industry, ${ }^{4}$ drying plays a significant role. From a methodological point of view, approaches and techniques are described in other domains related to thin film drying. Thermogravimetric analysis (TGA), ${ }^{5}$ inverse microRaman spectroscopy (IMRS), ${ }^{6}$ and a FTIR-spectroscopic $\operatorname{method}^{7}$ are often-cited techniques.

In the manufacturing of catalyst-coated membranes (CCMs) and membrane electrode assemblies for fuel cells or electrolyzers, the drying of generated wet catalyst layers is one process step. The investigation of this step is rarely documented in the literature. ${ }^{8-11}$ In the few available studies, the influence of the drying process on the product is demonstrated by weight measurements. For meaningful research activities in electrode drying, chemical selective measuring instruments are required. In addition to that, the method should not focus on microscopic areas, but it must provide information about the full area of the electrode active area.

In this study, a novel method is introduced which provides entire sample and chemical information. Even though the manufacture of CCMs is our focus, the system is not restricted to this field at all. The evaporated solvent content is transported by a gas flow to an FTIR spectrometer. The instrument analyzes the gas composition as a function of time. These data will be used to recalculate the residual dispersion composition at any time during the drying process. FTIRspectroscopy is used due to the high IR-activity of the solvents used. In comparison with standard IR-spectroscopy, the speed and signal-to-noise ratio are increased. In the past, the drying of the entire material to be dried was already analyzed by IRspectroscopy. ${ }^{12}$

In this work, the confidence level of the method is investigated. It focuses on developing procedures to estimate the precisions of devices which are used in the setup. This should result in an improvement of the measurement precision. The assumptions and estimations are checked by validation. Finally, the confidence level is analyzed to find the biggest influencing factor. For calculation and wording, ISO 5725 definitions and recommendations are used.

\section{METHODS}

In this part, the equipment is designated and the technical setup is illustrated. Furthermore, the evaluation principle is explained beginning with the recording of the raw data up to the recalculation of the sample composition. Additionally, the device-specific precision is discussed.

\section{A. Setup and materials}

The system is equipped with a drying path and a dilution path. In the drying path, the gas overflows the sample in the two-part drying chamber (inner dimensions: length $=160 \mathrm{~mm}$, width $=80 \mathrm{~mm}$, and height $=20 \mathrm{~mm}$ ). It flows out of the chamber after being loaded by the evaporated solvent content. Afterwards, the mixture is diluted by the gas originating from the dilution path. The drying path can be supplied by either nitrogen 5.0 (Air Liquid) or air $(<1 \mathrm{ppm}$ residual air moisture), while the dilution path is always connected to nitrogen. In both paths, mass flow controllers (Brooks, USA, device type: $5850 \mathrm{~S}, 0-1 \mathrm{l} / \mathrm{min}$ and $0-51 / \mathrm{min}$ ) set the desired 


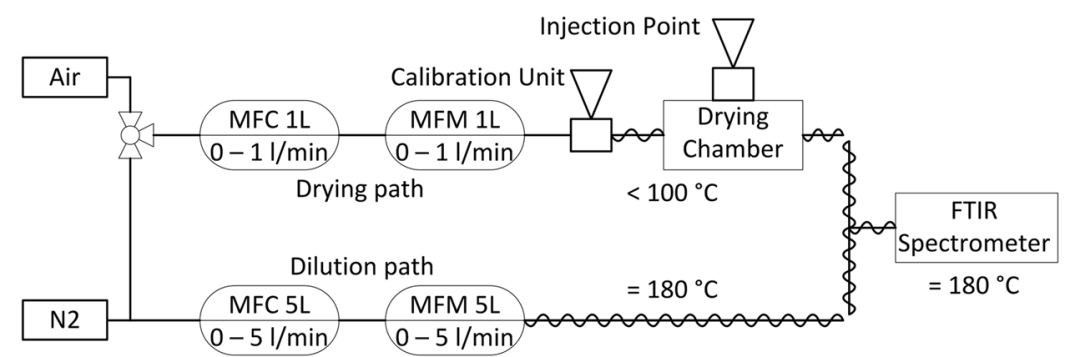

FIG. 1. Setup of the method. On the left-hand side, the drying gas flow enters the system. The top path is the drying path through the calibration unit and the drying chamber. The gas is set to the drying temperature. The bottom path is the dilution path. The gas temperature is controlled to be $180^{\circ} \mathrm{C}$. Both paths are combined after the drying chamber and regulated at $180^{\circ} \mathrm{C}$. The gas flows through the measuring cell of the FTIR-spectrometer and is then released from the system. gas flow rate. The flow is recorded by using mass flow meters (Analyt-MTC, Germany, device type: 358MLW, 0-1 1/min and $0-51 / \mathrm{min}$ ). The dilution allows an adjustable gas concentration which is detected by using the FTIR-spectrometer (Ansyco, Gasmet, Finland, device type: CX4000-FTIR, fixed path length: $10 \mathrm{~cm}$, resolution: $7.921 / \mathrm{cm}, 10$ spectra per second). The spectrometer uses Calcmet ${ }^{\mathrm{TM}}$ standard version for analysis. According to the recommended temperature for the analyzer, the gas temperature is controlled to be $180{ }^{\circ} \mathrm{C}$. The upper limit for the drying chamber and the gas temperature in the drying path is $100{ }^{\circ} \mathrm{C}$. The schematic is illustrated in Fig. 1, while a real picture is shown in Fig. 2.

Generally, samples can be placed on the base. This is shown in Fig. 3. The base and the lid of the drying chamber are pressed against a polytetrafluoroethylene (PTFE) gasket which is fitted in-between. The pre-tempered gas enters the drying chamber and is evenly distributed by a diffuser plate. The flow profile above the sample corresponds to a laminar flow. Afterwards, the gas leaves the drying chamber at the exit.

In the case of validation, droplets can be injected into the system by means of syringes (Hamilton, gastight, $1-5 \mathrm{ml}$ ). For this purpose, an injection site is in the drying chamber. Additionally, a window is implemented in the drying lid to visually observe the drying. A second injection site is installed in the drying path which is used for the calibration. The calibration is performed with the help of a dynamic calibration unit (Ansyco, Finland, Sycos K-DPG). This unit mainly consists of a syringe pump.

\section{B. Measuring principle}

The residual solvent content and the residual sample composition are the most interesting quantities of the evaluation.

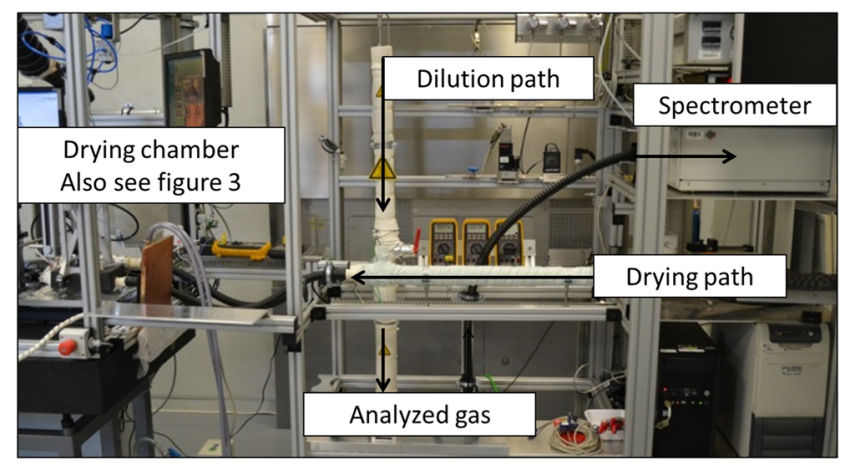

FIG. 2. Real picture of the test station with the drying and dilution gas flow direction.
Since an indirect measurement is used, these values are determined over several steps.

In a first step, the gas-phase composition is determined by using the FTIR-spectrometer. For this purpose, the absorbance of light, $A$, is detected. The absorbance is related to the path length of the light beam through the sample, $l=10 \mathrm{~cm}$, and to the volume fraction, $\phi_{i}$, of a substance i. By the use of a material- and wavelength-specific coefficient, $\varepsilon_{i, \lambda}$, called attenuation coefficient, Beer's law is obtained,

$$
A=\Sigma_{i} A_{i}\left(\phi_{i}, \varepsilon_{i, \lambda}\right)=l \Sigma_{i} \phi_{i} \varepsilon_{i, \lambda} .
$$

The absorbance is related to a background gas. In this method, nitrogen or air is used as the drying gas, and thus, the signal becomes zero for the pure corresponding gas. Since the background gas flow rate $V_{0}{ }^{\prime}$ is known, the gaseous volume flow of the substance $V_{i, g^{\prime}}$ can be related to its volume fraction in the total gas flow,

$$
\begin{gathered}
\phi_{i}\left(V_{i, g}{ }^{\prime}, V_{0}{ }^{\prime}\right)=V_{i, g}{ }^{\prime} /\left(V_{i, g}{ }^{\prime}+V_{0}{ }^{\prime}\right), \\
V_{i, g}{ }^{\prime}=\phi_{i} V_{0}{ }^{\prime} /\left(1-\Sigma_{i \neq 0} \phi_{i}\right) .
\end{gathered}
$$

Assuming an ideal gas with gas constant, $\mathrm{R}$, absolute temperature, $T$, pressure, $p$, and molar mass, $M_{i}$, of the substance, the volume fraction is converted into the drying mass flow rates $m_{i}{ }^{\prime}$,

$$
m_{i}{ }^{\prime}\left(\phi_{i}, V_{0}{ }^{\prime}, M_{i}\right)=p \phi_{i} V_{0}{ }^{\prime} M_{i} / R T\left(1-\Sigma_{i \neq 0} \phi_{i}\right) .
$$

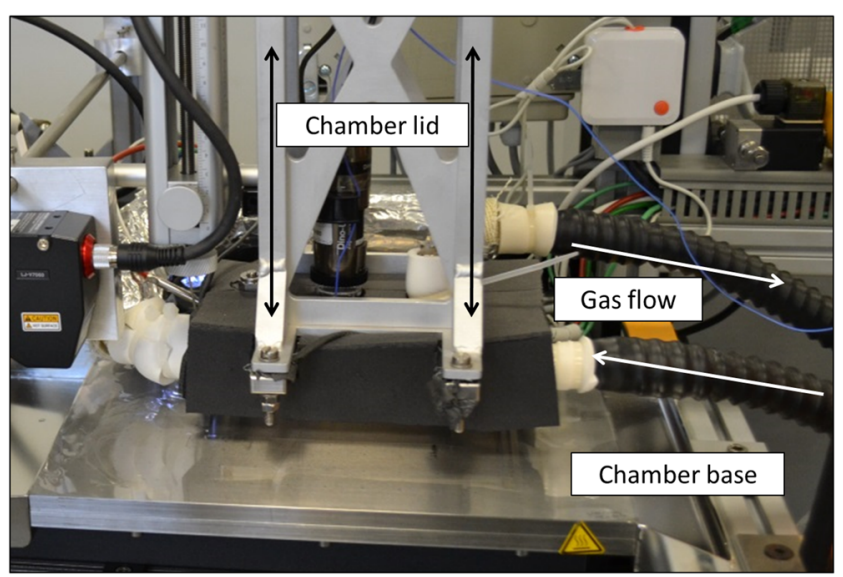

FIG. 3. Real picture of the drying chamber with a vertically movable (black arrows) lid. In the case of measuring, the lid is pressed against the base. A PTFE gasket is fitted in-between. The white arrows symbolize the direction of the gas flow. 
Furthermore, it is assumed that at the end of drying, all evaporation rates become zero $\left(m_{i}{ }^{\prime}=0\right)$. This means that no residual solvent content $\left(m_{r s c, i}=0\right)$ remains in the sample. Unfortunately, the exact moment a sample is completely dried cannot be determined reliably. Hence, an abort criterion is implemented into analysis. The recalculation of the residual solvent content starts at this point. The related time is described as $t_{l}<t_{d r y}$. The recalculation runs in the direction of earlier drying stages. The time of the first measurement point of the drying analysis is declared with $t_{f}$. The residual solvent mass content $m_{r s c, i}=m_{l, f, i}$ within an interval of analysis $\{l \leq j \leq f\}$ is a discrete function,

$$
m_{l, f, i}=\sum_{j=l}^{f}\left(t_{j}-t_{j-1}\right)\left(m_{j, i}{ }^{\prime}+m_{j-1, i}{ }^{\prime}\right) / 2 .
$$

The residual solvent composition $\omega_{r s c, i}$ is

$$
\omega_{r s c, i}=m_{r s c, i} / \Sigma_{i} m_{r s c, i} .
$$

This kind of evaluation meets the requirements made for the required method. The principle enables analyzing selectively the evaporation rates, and it provides information about the whole layer. However, the raw data are the spectral absorbance of the substances.

\section{Calibration of the spectrometer}

In accordance with Eq. (1), the spectral absorbance is converted into the volume fraction by Calcmet. Therefore, a calibration is needed that relates a certain absorbance to a volume fraction. This is performed by generating and recording test gases $\phi_{t g}=\phi_{i}$ of the known volume fraction. For this purpose, the background gas flow rate and the substance gas flow rate are adjusted according to Eq. (2). The liquid volume flow rate $V_{i, l}{ }^{\prime}$ of a substance $\mathrm{i}$ is convertible into the gaseous volume flow rate $V_{i, g}{ }^{\prime}$ by assuming an ideal gas,

$$
V_{i, g}{ }^{\prime}=\rho_{l} R T V_{i, l^{\prime}} / M_{i} p .
$$

The density of the liquid is designated with $\rho_{l}$. In this work, the background gas flow is kept constant and the volume flow of the substance to be calibrated is varied by the use of the calibration unit.

For each substance, several test gases of known volume fractions are generated. In the case of calibrating the spectrometer, these test gases are also called reference gases $\phi_{\text {ref }, i}(A)$. The related records are called reference spectra. In the range between two reference spectra, the relation between $\phi$ and A is interpolated. Additionally, this interpolation is correcting the non-linearity of Beer's law. This non-linearity results from the finite resolution of the spectrometer. The interpolated curve is designated with $\phi_{\text {int }}$. The range between $\phi_{\text {ref, } i}$ and $\phi_{r e f, i+1}$ including the interpolation is designated as the calibration curve $\phi_{C a l}$,

$$
\left[\phi_{\text {ref }, i}(A)<\phi_{\text {int }}(A)<\phi_{\text {ref }, i+1}(A)\right]=\phi_{C a l}(A) .
$$

Aside from pure solvents, generally, the spectrometer must also be calibrated for gas mixtures due to cross-sensitivity in the spectrum. In a first approach, this influence on the accuracy is neglected since the statistics from experimental results do not indicate a significant contribution.

\section{Methodology and error propagation}

The precise procedure of this correction as well as the actual precision of the interpolation is not accessible. The manufacturer specifies the deviation to be less than $2 \%$. According to the golden rule of measurement technology, ${ }^{13}$ this value seems to be too large. Therefore, an alternative procedure is necessary to check the validity of the interpolation procedure. Hence, for a calibration section, as shown in Eq. (8), additional test gases $\phi_{t g}$ are generated and analyzed by using the system. Under ideal conditions, $\phi_{t g}=\phi_{C a l}$ is found. If this assumption can be confirmed, the calibration will be accepted as sufficient. Otherwise, a correction must be implemented into the evaluation.

To compare $\phi_{t g}$ and $\phi_{C a l}$, a fitting process described by Press $^{14}$ is applied. The resulting fit is designated with $\phi_{\text {Cal.fit }}$,

$$
\phi_{C a l, f i t}\left(\phi_{t g}, a, b\right)=a \phi_{t g}+b .
$$

In Eq. (9), the fit parameters $a$ and $b$ symbolize the interpolation. If the deviation in the interpolation procedure $u\left(\phi_{\text {int }}\right)$ is negligible, it will be $a=1$ and $b=0$. However, in reality, test gases have uncertainties $u\left(\phi_{t g}\right)>0$ which may influence the fit parameters. The uncertainty is calculated according to the Guide to the Expression of Uncertainty in Measurement (GUM). ${ }^{15}$ The fit [Eq. (9)] is connected to the uncertainty $u\left(\phi_{\text {Cal }, f i t}\right)$. The covariance $\operatorname{Cov}(a, b)$ provides the correlation between the fit parameters. It is

$$
\begin{aligned}
u\left(\phi_{\text {Cal }, f i t}\right)= & {\left[\left(u\left(\phi_{t g}\right) a\right)^{2}+\left(u(a) \phi_{t g}\right)^{2}+u^{2}(b)\right.} \\
& \left.+2 \phi_{t g}{ }^{2} \operatorname{Cov}(a, b)\right]^{1 / 2} .
\end{aligned}
$$

Once again, it is obvious that the calibration uncertainty depends on the uncertainty of test gas generation and on the interpolation uncertainty.

As $u\left(\phi_{t g}\right)>0$, a reasonable estimation must be performed to determine $u\left(\phi_{t g}\right)$. In accordance with Eq. (2), the test gas generation depends on the background gas flow and on the substance gas flow. Hence, the precision of the test gas generation is limited to the precision of the mass flow meters and to the precision of the substance flow rate.

For an overview of the precisions, the manufacturer specifications are listed in Table I. They are related to $95 \%$ confidence interval, so, for normal distribution, $95 \%$ of the results are within twice the standard deviation.

It is worth checking the given values to determine whether they are correct. A procedure to improve information about the precision of the mass flow meter is rather complicated. In a first approach, the gas flow uncertainties given in Table I are interpreted as precision. This is performed since the actual meaning of accuracy is a non-quantifiable value. However, total background gas flow is determined by the volume flows in the drying path and in the dilution path [Eq. (11)]. No covariance appears between both paths due to a lack of correlation. The uncertainty in the gas flow rate is calculated with Eq. (12),

$$
\begin{gathered}
V_{0}{ }^{\prime}\left(V_{d r y}{ }^{\prime}, V_{d i l}{ }^{\prime}\right)=V_{d r y^{\prime}}+V_{d i l}{ }^{\prime}, \\
u\left(V_{0}{ }^{\prime}\right)=\left[u^{2}\left(V_{d r y}{ }^{\prime}\right)+u^{2}\left(V_{d i l}{ }^{\prime}\right)\right]^{1 / 2} .
\end{gathered}
$$


TABLE I. The specifications of devices and materials which are used for the test gas generation.

\begin{tabular}{lccc}
\hline \hline Flow & Device & Type & Quantity \\
\hline Liquid flow generation & KDS 100 (syringe pump) & Accuracy & $1 \%$ \\
& Hamilton gastight syringe & Accuracy & $1 \%$ nominal volume \\
& & Precision & $1 \%$ at $80 \%$ of the total volume \\
Background gas flow & Mass flow generation & Accuracy & $0.4 \%$ of reading \\
& & & $0.2 \%$ of full scale \\
\hline \hline
\end{tabular}

On the other hand, the liquid substance flow rate can be evaluated more precisely by weight measurement. Therefore, a syringe is filled with liquid water and it is fixed in the syringe pump. The feed rate is specified to a defined liquid volume flow. By this, the syringe is emptied into a porous medium. The porous medium prevents the generation of drops between the syringe and the absorbing material. This guarantees a steady liquid flow. However, fluctuations exist, but they are neglected in a first approach. The medium is weighted by using an analytical balance (Mettler Toledo, USA, device type: XSE205DU) over time. The procedure is repeated five times for different feed rates. Within each repetition, the signal is separated into $180 \mathrm{~s}$ intervals (designated with $i=1, \ldots, \mathrm{n}$ ). This time interval is chosen since it is the recording time when calibrating the spectrometer. The feed rate can deviate due to deviations both in the thread of the syringe stepped motor and in the inner diameter of the syringe. Further on, a slight fluctuation is expected since the repeatability of the syringe pump is limited by the manufacturer specification (Table I). Figure 4 illustrates this setup.

The mass, $m(t)$, will increase approximately linearly as a function of time with uncertainties $u(m)$ and $u(t)$. An estimate of the actual mass flow rate is obtained by fitting. The slope of the fit is the value of the estimated actual mass flow. The mass and mass flow rate are reconverted into the liquid volume and the liquid volume flow. The density fluctuation due to temperature variances is negligible. Subsequently, the fit for the $\mathrm{i}$-th $180 \mathrm{~s}$ interval is determined by the following equation:

$$
V_{a c t, i}\left(t, V_{a c t, i}{ }^{\prime}, V_{0}\right)=t V_{a c t, i}{ }^{\prime}+V_{0} \text {. }
$$

The variance of the estimated actual volume flow rate is assumed to be the standard error of the slope $s\left(V_{a c t, i}{ }^{\prime}\right)$.

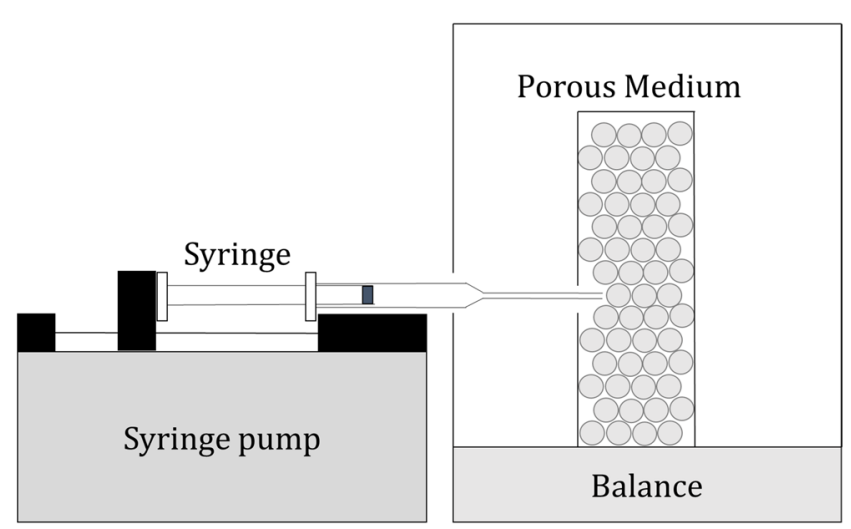

FIG. 4. Setup to determine the uncertainty of the liquid substance volume flow.
The mean standard error of all evaluations is designated with $s\left(V_{a c t}{ }^{\prime}\right)$. Further on, it is assumed that the mean slope of $\mathrm{n}$ evaluations $\left\{V_{a c t, 1}{ }^{\prime}, \ldots, V_{a c t, n^{\prime}}\right\}$ is the true value $V_{a c t}{ }^{\prime}$ with an uncertainty $u\left(V_{a c t}{ }^{\prime}\right)$ in its determination. Subsequently, the combined uncertainty for one $180 \mathrm{~s}$ interval is

$$
u_{c}\left(V_{a c t, i}{ }^{\prime}\right)=\left[s^{2}\left(V_{a c t}{ }^{\prime}\right)+u^{2}\left(V_{a c t}{ }^{\prime}\right)\right]^{1 / 2} \text {. }
$$

The set value is assumed to be deviating from the true value. The calibration of the set value is performed by fitting,

$$
\begin{aligned}
u_{c}\left(V_{\text {set }, f i t}{ }^{\prime}\right)= & {\left[\left(u_{c}\left(V_{a c t, i^{\prime}}\right) a\right)^{2}+\left(u(a) V_{a c t}{ }^{\prime}\right)^{2}\right.} \\
& \left.+u^{2}(b)+2 V_{a c t}{ }^{\prime 2} \operatorname{Cov}(a, b)\right]^{1 / 2} .
\end{aligned}
$$

The value of $u\left(V_{\text {set }, f i t}{ }^{\prime}\right)$ delivers the uncertainty of the syringe pump adjustment. It is assumed as the standard uncertainty $u\left(V_{i, l^{\prime}}\right)$.

After evaluating the precision of the liquid substance volume flow and background gas flow, the standard deviation of the test gas volume fraction is calculated. Therefore, Eq. (7) is used to convert the liquid volume flow into the gaseous volume flow. By the use of Eq. (2), the volume fraction is calculated. The related uncertainty is formulated by

$$
\begin{aligned}
u\left(\phi_{t g}\right)= & {\left[\left(-u\left(V_{i}{ }^{\prime}\right) V_{0}{ }^{\prime} /\left(V_{i}{ }^{\prime}+V_{0}{ }^{\prime}\right)^{2}\right)^{2}\right.} \\
& \left.+\left(u\left(V_{0}{ }^{\prime}\right) V_{i}{ }^{\prime} /\left(V_{i}{ }^{\prime}+V_{0}{ }^{\prime}\right)^{2}\right)^{2}\right]^{1 / 2} .
\end{aligned}
$$

This value can be used to check the calibration by inserting it into Eq. (10).

\section{E. Validation procedure}

The assumptions about the measurement precision need to be validated. This is performed by injecting a known mass of a substance into the system. For this purpose, the injection site of the drying chamber is used. If the deviation measured is less than the calculated uncertainty [Eq. (17)], the system will be considered to be working,

$$
u\left(\phi_{j}\right)=\left[u^{2}\left(\phi_{t g}\right)+u^{2}\left(\phi_{i n t}\right)+u^{2}\left(\phi_{V_{-} 0^{\prime}}\right)\right]^{1 / 2} .
$$

Equation (17) is the measurement uncertainty for a single data point $\mathrm{j}$. It consists of the uncertainties of the volume flow, $u^{2}\left(\phi_{V_{-}}{ }^{\prime}\right)$, and the calibration. The calibration is divided into the uncertainties of test gas generation, $u^{2}\left(\phi_{t g}\right)$, and interpolation $u^{2}\left(\phi_{\text {int }}\right)$. The calculation of the relative deviation is easy to calculate for constant time steps. If the time steps were different, the integral of Eq. (17) would need to be solved over time. This integral will also be necessary if the uncertainty is needed as an absolute value as a function of time. However, the relative measurement uncertainty $u_{r}\left(\varphi_{j}\right)$ for a whole measurement 
is calculated according to the following equation:

$$
u_{r}\left(\phi_{j}\right)=\Sigma_{j} \mathrm{u}\left(\phi_{j}\right) / \Sigma_{j} \phi_{j}
$$

\section{EXPERIMENTAL}

This section consists of five parts. First, the precision of the test gas generation is determined. By means of this information, the interpolation procedure is checked in the second part. In the third part, the resulting total measurement deviation is validated experimentally. The repeatability is checked in the fourth part. Finally, an analysis of the resulting measurement precision is performed to evaluate future work packages.

\section{A. Precision of test gas generation}

As mentioned, the manufacturer specification is used for the precision of the gas flow (see Table I). For test gas generation, the background gas flow is set to $V_{d r y}{ }^{\prime}=0.5 \mathrm{ml} / \mathrm{h}$ and $V_{d i l}{ }^{\prime}=1.5 \mathrm{ml} / \mathrm{h}$. These are the values which will be used in the validation part. In accordance with Eq. (12), the standard uncertainty of background gas flow is $7.28 \mathrm{ml} / \mathrm{h}$.

The evaluation of the substance volume flow rate precision was performed using multiple syringes to generate a statistically significant set of liquid flow rates. As an example, feed rates of $1.2,2.4$, and $3.6 \mathrm{ml} / \mathrm{h}$ were produced using a $1 \mathrm{ml}$ syringe. Using Eq. (2), the combination of the background gas flow rate $(1.5 \mathrm{l} / \mathrm{min})$ and the feed rate interval $[0 ; 3.6 \mathrm{ml} / \mathrm{h}]$ results in volume fractions of $[0 ; 4.73 \mathrm{vol} . \%]$ for water, [0;1.16 vol. \%] for isopropyl alcohol, and [0;1.21 vol. \%] for acetone. This is the covered calibration range of the spectrometer.

An exemplary set of liquid flow rate measurement data is discussed in the following. The target feed rate is chosen to be $1.20 \mathrm{ml} / \mathrm{h}$. The data set consists of 50 measurements of $180 \mathrm{~s}$. The residuals between the weight measurements and the linear fit are normally distributed. This means that no significant drift can be observed neither along the thread of the syringe stepped motor nor in the syringe diameter

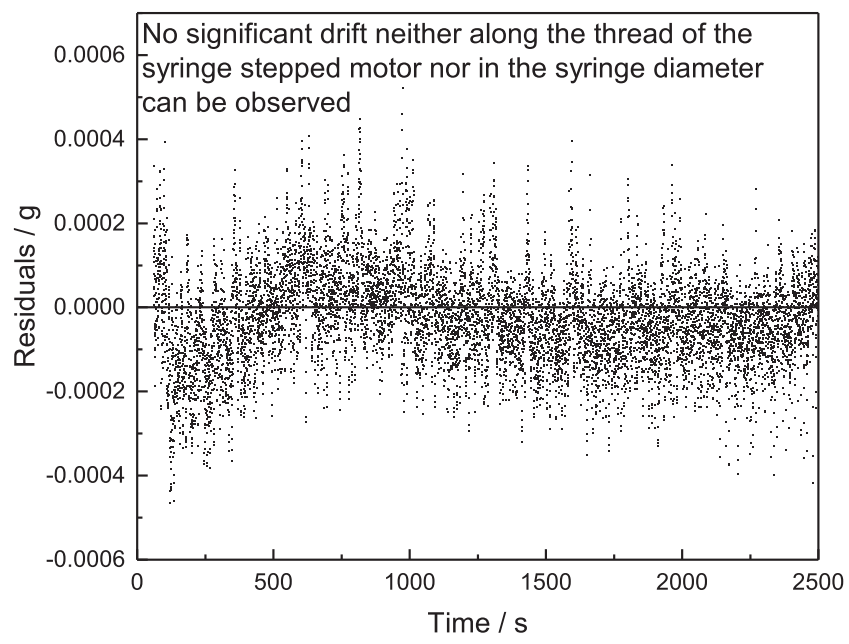

FIG. 5. Illustration of the mass residuals between the fit and actual mass for an exemplary feed rate of $1.20 \mathrm{ml} / \mathrm{h}$. (see Fig. 5). The mean uncertainty within each measurement is $s\left(V_{a c t}{ }^{\prime}\right)=0.0003 \mathrm{ml} / \mathrm{h}$. For the whole set, $V_{a c t}{ }^{\prime} \pm u\left(V_{a c t}{ }^{\prime}\right)$ $=1.1961 \pm 0.0020 \mathrm{ml} / \mathrm{h}$ is determined for the true value and its uncertainty. The value of $u\left(V_{a c t}{ }^{\prime}\right)$ is in the range 0.0015 $-0.0020 \mathrm{ml} / \mathrm{h}$ for all set feed rates. For error propagation, a conservative value of $0.0021 \mathrm{ml} / \mathrm{h}$ is assumed. The actual liquid flow rates are approached to be normally distributed (see Fig. 6). Subsequently, the combined uncertainty of the liquid volume flow for one $180 \mathrm{~s}$ interval is determined to be $u_{c}\left(V_{a c t}{ }^{\prime}\right)=0.0021 \mathrm{ml} / \mathrm{h}$. The measurements were carried out for water. However, all results are calculated as volume flows. Therefore, the data are valid for all substances.

This evaluation is carried out for different liquid volume flow rates. Fitting these data in accordance with Eq. (15) results in the following equation:

$$
V_{\text {set }, \text { fit }}{ }^{\prime}=1.0022 V_{\text {act }}{ }^{\prime}-0.0012 \mathrm{ml} / \mathrm{h} \text {. }
$$

The corresponding uncertainty in the fit is $u\left(a_{V^{\prime}}\right)=8.65$ $\times 10^{-5} \mathrm{ml} / \mathrm{h}$ for the slope parameter and $u\left(b_{V^{\prime}}\right)=1.70 \times 10^{-4}$ $\mathrm{ml} / \mathrm{h}$ for the offset parameter. The covariance is $\operatorname{Cov}\left(a_{V^{\prime}}, b_{V^{\prime}}\right)$ $=1.34 \times 10^{-8} \mathrm{ml} / \mathrm{h}$. Concerning the fit and the uncertainties, the offset parameter deviates from 0 , while the slope parameter deviates from 1 . Therefore, the values must be adjusted by the calibration. Equation (16) determines the uncertainty in the set liquid substance volume flow to be $u\left(V_{i, l^{\prime}}\right)=u_{c}\left(V_{\text {set } f t^{\prime}}{ }^{\prime}\right)$ $=0.0021 \mathrm{ml} / \mathrm{h}$ for a true feed rate of $1.20 \mathrm{ml} / \mathrm{h}$.

The evaluation of the standard deviation, $u\left(\phi_{t g}\right)$, depends on the molar mass of the substance to be calibrated [see Eq. (7)]. An increase in the molar mass results in a smaller gaseous volume flow. Consequently, the volume fraction of a test gas is lower at the same syringe pump feed rate. Therefore, the uncertainty in the feed rate has a bigger influence at the same volume fraction. The meaning of the results for water and isopropyl alcohol test gases is illustrated in Fig. 7.

\section{B. Checking of interpolation procedure}

Reference spectra are recorded for isopropyl alcohol at $1 \%$ and $2 \%$ volume fraction in nitrogen. Afterwards, the Calcmet software runs the interpolation procedure [see

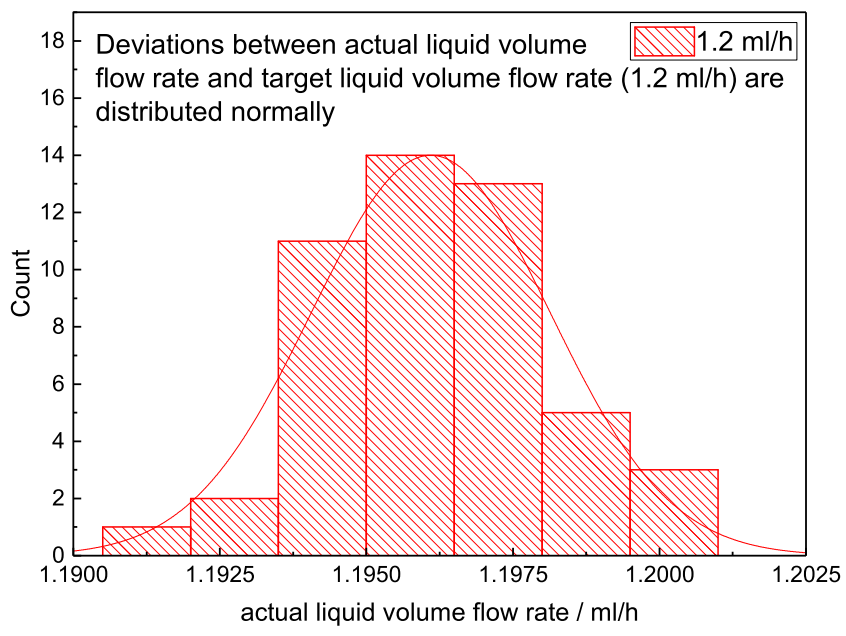

FIG. 6. Illustration of the actual liquid volume flow rate. The set value is 1.20 $\mathrm{ml} / \mathrm{h}$. In a first approach, the distribution is processed as normally distributed. 


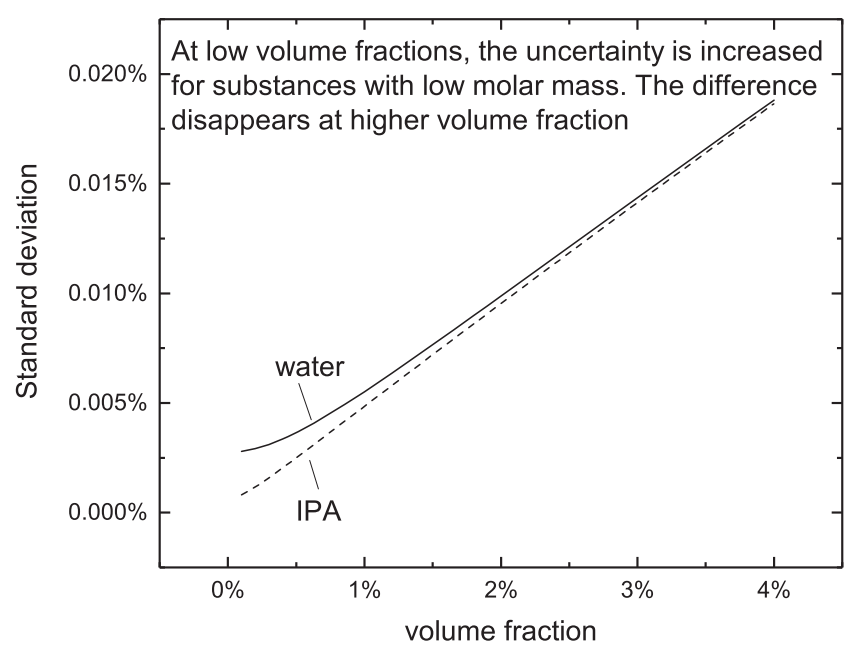

FIG. 7. The uncertainty is illustrated as the standard deviation of the volume fraction of a test gas. The values are referred to the mean of a $180 \mathrm{~s}$ calibration process and to different volume fractions.

Eq. (8)]. Test measurements must confirm the volume fractions resulting from the calibration. The test gases $\phi_{\mathrm{tg}}$ are generated by calculating the feed rate $V_{i, g}$ in accordance with Eq. (3). The volume fractions are chosen to be between $0.3 \%$ and $1.3 \%$. The resulting gaseous flow is converted into the liquid substance volume flow $V_{i, l^{\prime}}$ by Eq. (7). The resulting value is inserted as the actual liquid volume flow into Eq. (19) to obtain the set feed rate $V_{\text {set,fit }}{ }^{\prime}$ which is adjustable in $10 \mu \mathrm{l} / \mathrm{h}$ steps only. Therefore, the result needs to be rounded and reconverted into the true feed rate. This feed rate again has to be converted back into the gaseous substance volume flow $V_{i, g}{ }^{\prime}$ by Eq. (7). By means of Eq. (2), the target volume fraction of the test gas is determined.

The indicated values are determined by measurements. The measurements are continued for at least 50 data points for $1.3 \%$ volume fraction and 175 data points for $0.3 \%$ volume fraction. These numbers are limited by the syringe volume and the chosen feed rate. The average and standard errors of the data sets provide the values for fitting in

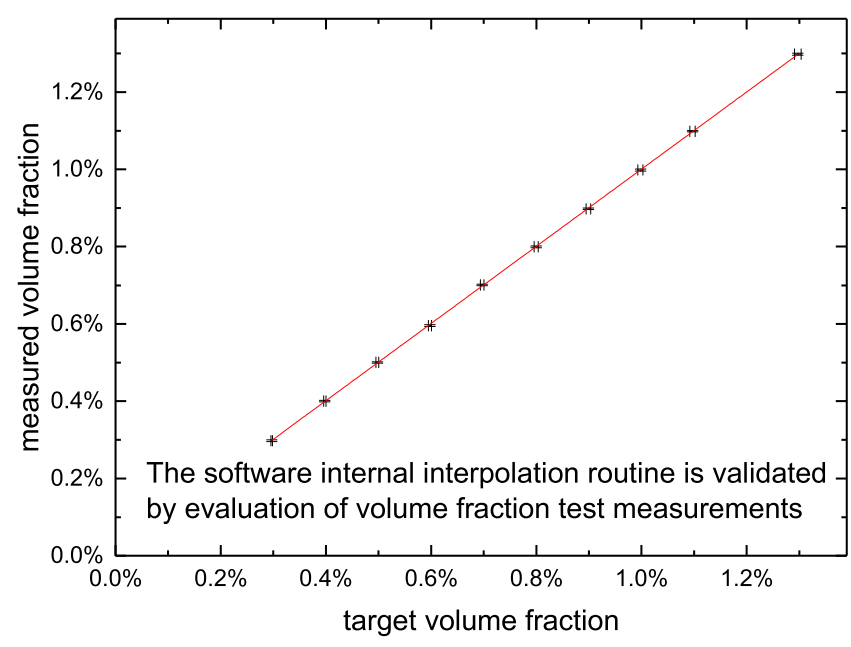

FIG. 8. The measured volume fraction is illustrated as a function of the target volume fraction. The red line illustrates the fit. accordance with Eq. (9). The slope is evaluated to be 0.998 \pm 0.005 (Fig. 8). This value enables the assumption $u\left(\phi_{\text {int }}\right)$ $\cong 0$, meaning that the interpolation is validated, and thus, $u\left(\phi_{t g}\right)=u\left(\phi_{\text {Cal }}\right)$.

\section{Validation}

For validation, five isopropyl alcohol and water solutions are injected as droplets into the drying chamber. As a substrate, glass fiber reinforced PTFE is used. The droplets are not wetting the PTFE; thus, they remain in the droplet form with a radius less than $10 \mathrm{~mm}$, while they start drying after injection instantly. As an example, Figs. 7 and 8 in this section illustrate the $62.30 \%$ isopropyl $/ 37.70 \%$ water mixture. For all tests, the drying temperature is $50{ }^{\circ} \mathrm{C}$ and the gas flow rate is set to $V_{d r y}{ }^{\prime}=0.5 \mathrm{ml} / \mathrm{h}$ and $V_{d i l}{ }^{\prime}=1.5 \mathrm{ml} / \mathrm{h}$. The abort criterion is $\phi\left(t_{l}\right)<0.01 \%$ for isopropyl alcohol and $\phi\left(t_{l}\right)<0.035 \%$ for water. An extrapolation of the mass flow rates delivers an estimate for the residual mass. It is determined to be $m\left(t_{d r y}>t_{l}\right)=1 \mathrm{mg}$ and $u\left(m\left(t_{d r y}>t_{l}\right)\right) \cong 1 \mathrm{mg}$. This value is considered in the data. By weight comparison of the PTFE substrate before and after the drying process, it is guaranteed that the total mass injected leave the system by drying. By the use of Eq. (4), the mass flow rates are evaluated. The exemplary mass flow rate as a function of time is shown in Fig. 9.

In accordance with Eq. (5), the residual solvent content is calculated. The result is illustrated as a function of time in Fig. 10. The residual solvent composition is finally recalculated with Eq. (6), and it is illustrated in Fig. 11.

Table II shows the injected target mass as well as the actual mass detected for all five samples. In addition to that, the relative deviation is specified.

Finally, the deviations declared in Table II are compared with the expected deviations which result from Eq. (19). The values are illustrated in Fig. 12. According to recommendation, ${ }^{16}$ the $95 \%$ confidence interval is used. For normally distributed random values, this is twice the standard

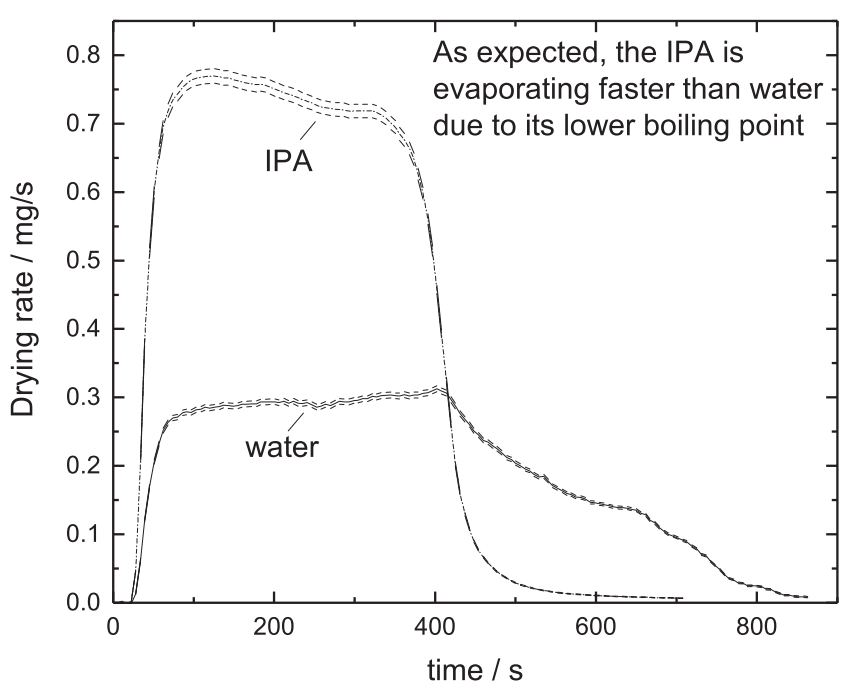

FIG. 9. The drying rate is plotted as a function of time. The isopropyl alcohol evaporating rates are higher in early drying stages. After $400 \mathrm{~s}$, the drying selectivity is reversed. After $700 \mathrm{~s}$, no reliable alcohol signal is obtained any more. 


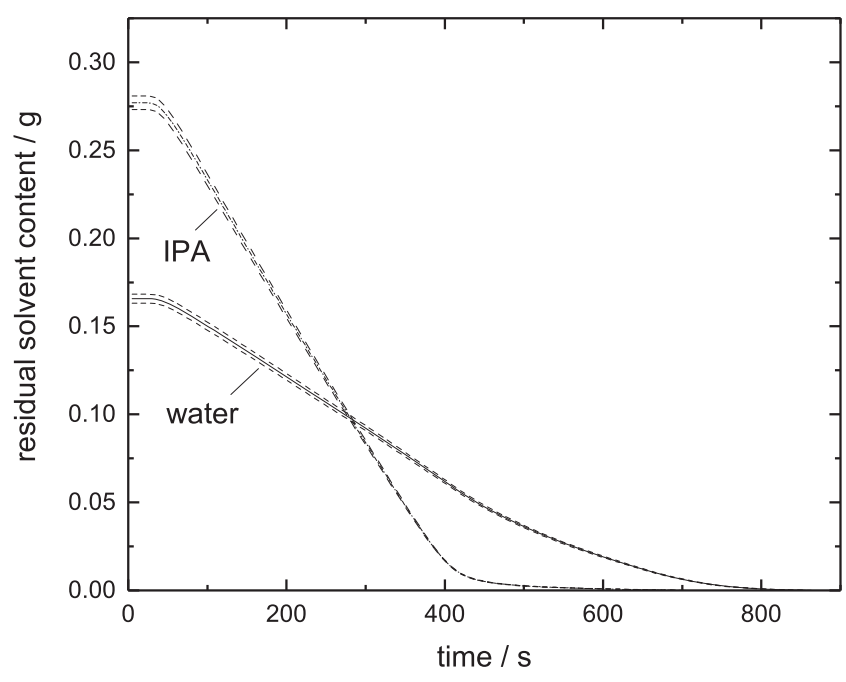

FIG. 10. The figure illustrates the residual solvent content as a function of time for water and isopropyl alcohol in the sample. The initial mass of isopropyl alcohol is higher than the initial mass of water. However, in compliance with Fig. 9, after $700 \mathrm{~s}$ of drying, the residual liquid in the sample is only water.

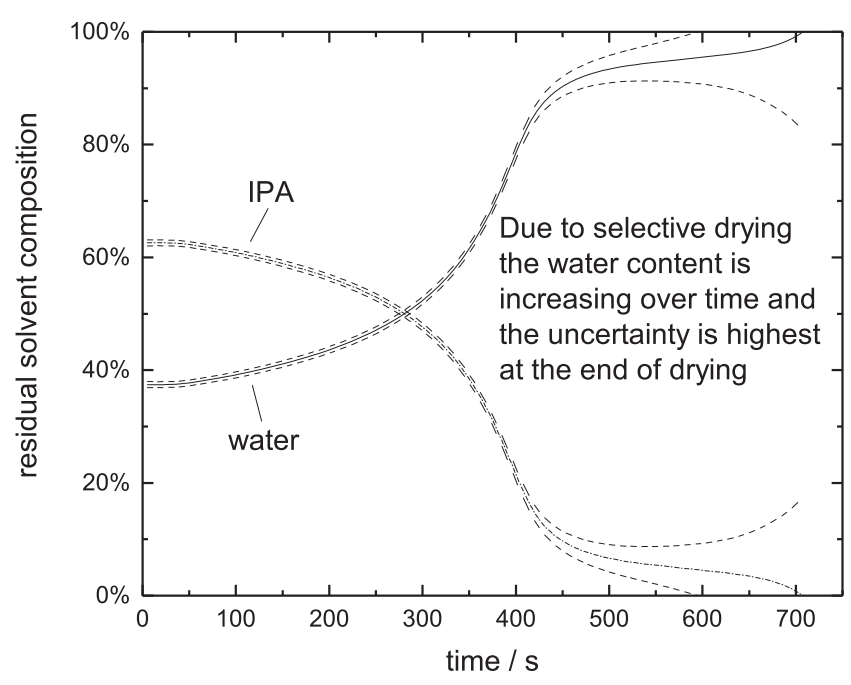

FIG. 11. The residual solvent composition is illustrated as a function of time. In accordance with Fig. 8, the initial composition consists of more isopropyl alcohol than water. During the drying process, the composition is reversed. The mass fraction of water in the sample increases to $100 \mathrm{wt}$ \% until the end of drying.

deviation. Obviously, the precision calculation is an appropriate estimation for the deviations.

\section{Repeatability}

In the case of Table II, the deviations are spreading between $-0.68 \%$ and $+1.70 \%$ for various mixtures. Aside from this, the repeatability also rates the quality of measurement. Therefore, an isopropyl alcohol and water solution is injected ten times to obtain the test-retest-reliability. The mass fraction of isopropyl alcohol is $\omega=78.19 \%$, and the total injected mass is $m=0.42005 \pm 0.00080 \mathrm{~g}$. The gas flow rates are $V_{d r y}{ }^{\prime}=0.5 \mathrm{ml} / \mathrm{h}$ and $V_{d i l}{ }^{\prime}=1.5 \mathrm{ml} / \mathrm{h}$, and the drying temperature is $T=60^{\circ} \mathrm{C}$.

The test-retest precision is significantly higher than the general explainable measurement precision (Fig. 13). In this
TABLE II. Target mass (index tar) and actual mass detected (index act) for isopropyl alcohol (IPA) and water $\left(\mathrm{H}_{2} \mathrm{O}\right)$. The relative deviation measured is given as $\mathrm{dm} / \mathrm{m}$.

\begin{tabular}{lccc}
\hline \hline$\omega(\mathrm{IPA})(\%)$ & $\mathrm{m}(\mathrm{IPA})_{\mathrm{tar}} / \mathrm{g}$ & $\mathrm{m}(\mathrm{IPA})_{\mathrm{act}} / \mathrm{g}$ & $\mathrm{dm} / \mathrm{m}$ \\
\hline 100 & 0.1996 & 0.2006 & $+0.28 \%$ \\
62.30 & 0.2733 & 0.2780 & $+1.70 \%$ \\
49.93 & 0.1844 & 0.1873 & $+1.56 \%$ \\
29.56 & 0.1014 & 0.1023 & $-0.68 \%$ \\
16.47 & 0.0570 & 0.0579 & $+1.70 \%$ \\
\hline$\omega(\mathrm{IPA})(\%)$ & $\mathrm{m}\left(\mathrm{H}_{2} \mathrm{O}\right)_{\mathrm{tar}} / \mathrm{g}$ & $\mathrm{m}\left(\mathrm{H}_{2} \mathrm{O}\right)_{\mathrm{act}} / \mathrm{g}$ & $\mathrm{dm} / \mathrm{m}$ \\
\hline 100 & 0 & 0 & 0 \\
62.30 & 0.1654 & 0.1667 & $+0.82 \%$ \\
49.93 & 0.1850 & 0.1865 & $+1.45 \%$ \\
29.56 & 0.2415 & 0.2450 & $+0.39 \%$ \\
16.47 & 0.2916 & 0.2926 & $+0.34 \%$ \\
\hline \hline
\end{tabular}

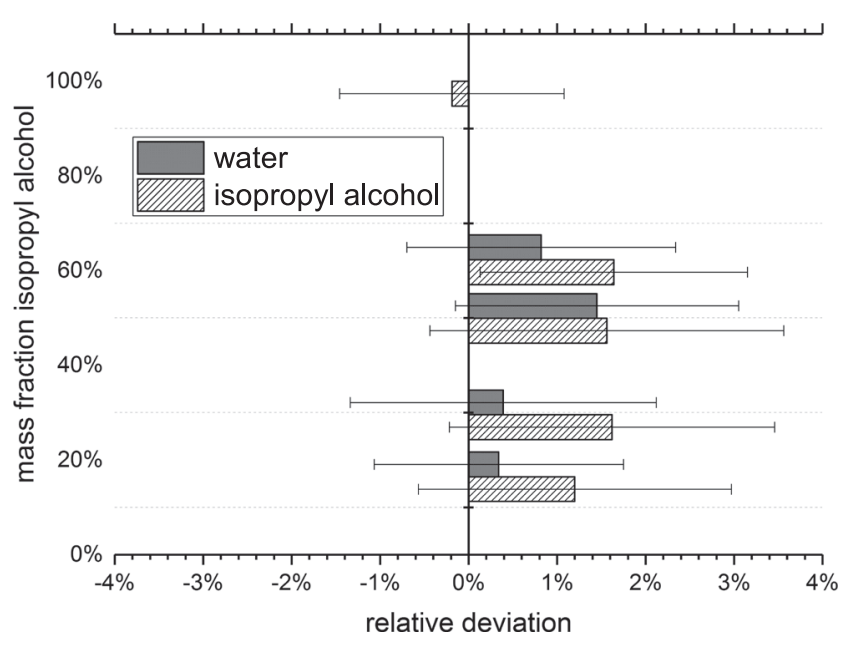

FIG. 12. This figure illustrates the relative deviation between the measured masses and the initial mass injected into the system. The data are derived from Table II. The measurement uncertainty is symbolized by the error bars. Except from the $62.30 \mathrm{wt}$. \% isopropyl measurement, the data match the expectations from calculation.

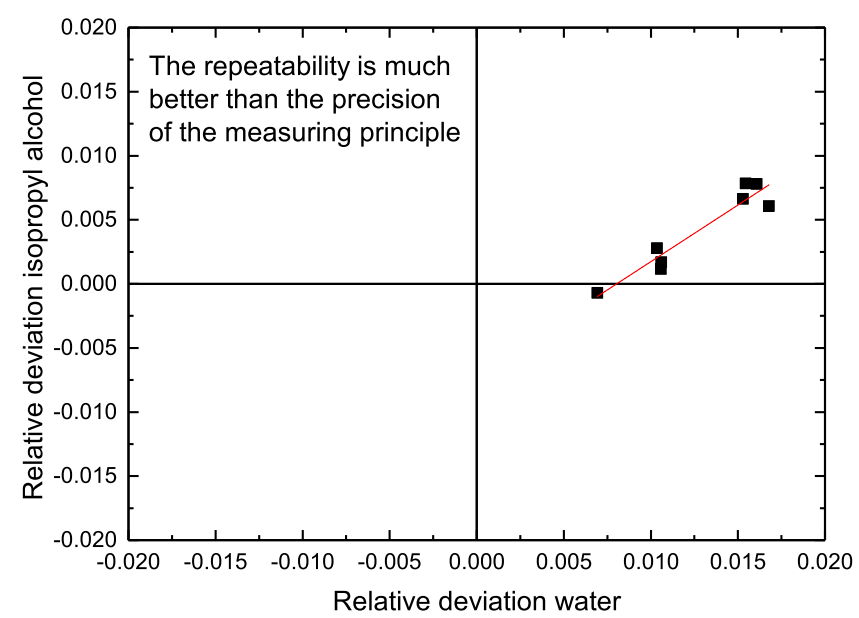

FIG. 13. The relative deviation of isopropyl alcohol is illustrated as a function of the relative deviation of water. The scale of the diagram is the range of the values obtained by error propagation. 
case, the standard errors of the deviations measured are $0.36 \%$ for water and $0.33 \%$ for isopropyl alcohol. The repetitive measurements indicate a strong dependence between the deviations. The coefficient of determination is $R^{2}=0.91$. Obviously, the deviations are influenced similarly. The reason for this cannot be explained in a verifiable way. However, the influence seems to be not selective; thus, two options appear to be traceable. On the one hand, the precision of the target mass injection could be worse than expected. In fact, this effect should be $0.05 \%$ which is neglected in the precision calculation. On other hand, the repeatability of the gas flow rate may be worse than expected. The standard deviations of the gas flow rates that are recorded may explain a relative deviation of $0.1 \%$. In both cases, the mass fractions are the same, but the actual injected mass differs from the assumed mass.

\section{E. Analysis of precision calculation}

The evaluation of the measurement precision is needed to quantify the reliability of the measurement. In addition, this value can be analyzed. If the precision of the system should be improved, the analysis will define the best approach for this issue. Therefore, the sources of deviations are extracted from the data.

For the example from the validation part, the squared fraction of the explainable substance flow rate deviation is illustrated in Fig. 14. A value below 50\% signalizes that the background gas flow uncertainty has a bigger impact on the precision than the uncertainty in the substance flow rate. The substance flow rate is part of the calibration, while the background gas flow is part of both calibration and measurement [see Eq. (17)]. In the case of values above 50\%, the precision of the substance flow rate limits the total measurement precision. The equality volume fraction depends on the substance. For water, this point is $0.40 \%$, and for isopropyl alcohol, it is $0.16 \%$. Hence, an improvement of the precision strongly depends on the volume fractions which should be

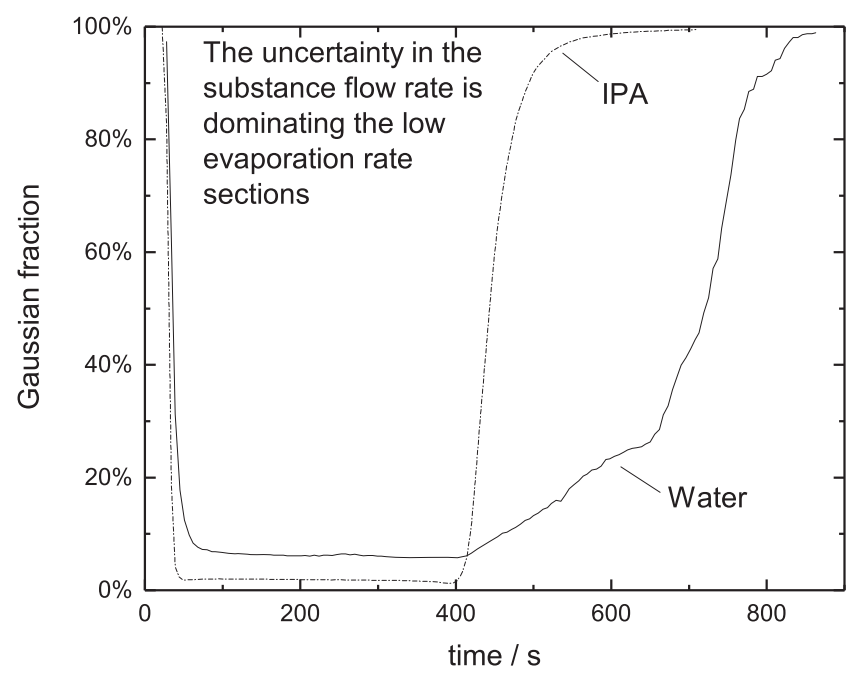

FIG. 14. The diagram represents the squared fraction (Gaussian fraction) of the substance flow precision on the total measurement uncertainty. In compliance with Figs. 5 and 7, at low volume fractions, the uncertainty in the substance volume flow is more important than the background gas volume flow. detected. However, increasing the precision of the gas flow rate seems to be preferable for the current application. This can be explained by calculation [Eq. (16)]. The volume fractions are below 5\%; thus, $V_{0}{ }^{\prime} \gg V_{i, g}{ }^{\prime}$. Therefore, an increasing substance flow rate does not change significantly the derivative in the direction of $V_{i, g}{ }^{\prime}$. Hence, the influence of the substance flow rate on the precision of the volume fraction is a constant. On the other hand, the derivative in the direction of $V_{0}{ }^{\prime}$ increases approximately linearly with increasing substance flow rates. Consequently, the explainable deviations resulting from the gas flow rate increase proportional to the volume fraction. These facts are visible in Fig. 9. While the confidence level is enlarged for high isopropyl alcohol flow rates, it becomes invisible for lower flow rates. Therefore, it is more efficient to improve the background gas flow rate precision.

\section{CONCLUSION}

The method presented here meets the requirements to analyze drying processes of electrodes. The drying curves consider the evaporation of the whole layer instead of local positions. Additionally, the method enables measuring the selectivity of drying and evaluating the time-dependent composition of samples. Therefore, this instrument is a significant improvement of existing methods to analyze electrode drying.

In addition, the method enables a precise investigation of drying processes. The expected deviations were calculated to be less than $2 \%$. This was validated by measurements for several isopropyl alcohol/water mixtures. Within the concentrations discussed, the internal interpolation of Calcmet was rated to be better than that specified by the manufacturer. The precision of the syringe and of the syringe stepped motor was analyzed in combination. It was found that the precision of liquid flow rates is significantly better than that indicated in data sheets. The repeatability of the system is much better than the general precision. The value was quantified to be $<0.40 \%$. Finally, from uncertainty analysis, it became obvious that improving the background gas flow rate precision is preferable in the future. This was justified by calculation.

The precision of measuring $1 \%$ volume fraction water in background gas should be $3.44 \%$ according to the manufacturer specifications. This value was decreased to $1.48 \%$ by analyzing the true precisions of the devices used.

\section{ACKNOWLEDGMENTS}

The authors acknowledge financial support by the Bavarian Ministry of Economic Affairs and Media, Energy and Technology for the joint projects in the framework of the Helmholtz Institute Erlangen-Nürnberg for Renewable Energy (IEK-11) of Forschungszentrum Jülich.

\footnotetext{
${ }^{1}$ F. Font, B. Protas, G. Richardson, and J. Foster, e-print arXiv:1801.01580 [cond-mat.soft] (2018).

${ }^{2}$ B. Schmidt-Hansberg, H. Colsmann, U. Lemmer, and W. Schabel, Eur. Phys. J.: Spec. Top. 166, 49 (2009).
} 
${ }^{3}$ S. Schrank, B. Kann, E. Saurugger, M. Hainschitz, M. Windbergs, B. J. Glasser, J. Khinast, and E. Roblegg, Int. J. Pharm. 478, 779 (2015).

${ }^{4}$ A. Calín-Sánchez, A. Kharaghani, K. Lech, A. Figiel, A. CarbonellBarrachina, and E. Tsotsas, Food Bioprocess Technol. 8, 63 (2015).

${ }^{5}$ B. D. Barr-Howell, D. Barbara, and E. J. Gordon, Thermochim. Acta 180, 147 (1991).

${ }^{6}$ W. Schabel, P. Scharfer, M. Muller, I. Ludwig, and M. Kind, Chem. Ing. Tech. 75, 1336 (2003).

${ }^{7}$ R. Saure, G. R. Wagner, and E. U. Schlunder, Surf. Coat. Technol. 99, 257 (1998).

${ }^{8}$ T. Munekata, T. Suzuki, S. Yamakawa, and R. Asashi, Phys. Rev. E 88, 052314 (2013).

${ }^{9}$ T. Suzuki, M. Kobayashi, H. Tanaka, M. Hayase, and S. Tsushima, ECS Trans. 69, 465 (2015).
${ }^{10}$ T. Suzuki, S. Tsushima, H. Tanaka, and M. Hayase, Int. J. Hydrogen Energy 41, 20236 (2016).

${ }^{11}$ J. Wang, M.S. thesis, University of Waterloo, 2015.

${ }^{12}$ H. Groenewold and E. Tsotsas, Chem. Eng. Sci. 62, 481 (2007).

${ }^{13} \mathrm{~J}$. Wang, "Formation and Characteristics of Microstructures from PEM Fuel Cell Catalyst Ink Drying," M.S. thesis, UWSpace, 2015, see http://hdl.handle.net/10012/9537.

${ }^{14}$ W. H. Press, Numerical Recipes: The Art of Scientific Computing, $785 \mathrm{ff}$ (University Press, Cambridge, 2007).

${ }^{15}$ JCGM104:2009, "Evaluation of measurement data-An introduction to the 'Guide to the expression of uncertainty in measurement' and related documents," (JCGM, 2009).

${ }^{16}$ JCGM106:2012, "Evaluation of measurement data-The role of measurement uncertainty in conformity assessment," (JCGM, 2012). 